\title{
EFFECT OF WATER, NUTRIENT AND WEED MANAGEMENT ON THE YIELD AND QUALITY OF AROMATIC BORO RICE (CV. BRRI dhan50)
}

\author{
S.K. Paul*, M.C. Ray, M.A.R. Sarkar and S.K. Sarkar \\ Department of Agronomy, Bangladesh Agricultural University, Mymensingh 2202, Bangladesh \\ *Corresponding E-mail: skpaul@bau.edu.bd
}

(Received: 25 April 2019, Accepted: 30 July 2019)

Keywords: Yield, grain protein, aromatic Bororice

\begin{abstract}
An experiment was conducted at the Agronomy Field Laboratory, Bangladesh Agricultural University, Mymensingh during January to June 2016 to observe the effect of water, nutrient and weed management practices on the yield and quality of aromatic Boro rice (cv. BRRI dhan50). The experiment consisted of three water managements viz. conventional flood irrigation, AWD (Alternate Wetting and Drying) and SRI (System of Rice Intensification); two nutrient managements viz. recommended dose of inorganic fertilizers (Urea, TSP, MoP, Gypsum, $\mathrm{ZnSO}_{4} @ 250,120,120,100,10 \mathrm{Kg} \mathrm{ha}^{-1}$, respectively) and 25\% less than recommended dose of inorganic fertilizer + poultry manure @ $2.5 \mathrm{t}$ $\mathrm{ha}^{-1}$; and three weed managements viz. weedy check (control), two hand weeding at 20 and 40 DAT, and pre-emergence herbicide (Rifit 33EC) followed by post emergence herbicide (Fast klin 10WP) application. The highest number of effective tillers hill ${ }^{-1}$, grains panicle ${ }^{-1}$,grain yield and protein (\%) in grain were obtained in SRI water management which was followed by AWD and conventional flood irrigation. Between the two nutrient management practices, application of $25 \%$ less than recommended dose of inorganic fertilizer + poultry manure @ $2.5 \mathrm{t} \mathrm{ha}^{-1}$ produced higher number of effective tillers hill ${ }^{-1}$,grains panicle ${ }^{-1}, 1000$-grain weight, grain yield and protein (\%) in grain than in organic fertilizer. Among the weed management practices, application of pre-emergence herbicide (Rifit 33EC) followed by post-emergence herbicide (Fast Klin 10WP) produced the highest number of effective tillers hill ${ }^{-1}$,grains panicle ${ }^{1}, 1000$-grain weight, grain yield and protein (\%) in grain followed by two weedings at 20 and 40 DAT while the lowest grain yield was obtained in weedy check. The highest number of effective tillers hill ${ }^{-1}$,grains panicle 1 , grain yield and protein (\%) in grain was found in SRI water management combined with 25\% less than recommended dose of inorganic fertilizer + poultry manure @ $2.5 \mathrm{t} \mathrm{ha}^{-1}$ and application of preemergence herbicide followed by post-emergence herbicide. So, it may be concluded that, to get the highest grain yield in aromatic Boro rice, SRI method along with application of $25 \%$ less than recommended dose of inorganic fertilizer + poultry manure @ $2.5 \mathrm{t} \mathrm{ha}^{-1}$ and applying preemergence herbicide (Rifit 33EC) followed by post emergence herbicide (Fast Klin 10WP) could be recommended.
\end{abstract}

\section{Introduction}

Rice (Oryza sativa) is the most extensively cultivated cereal crop in Bangladesh. Aromatic rice contributes a small portion (10\%) but an important subgroup of rice production. In recent years, aromatic rice has been introduced to the global market because of its taste, deliciousness and high price (Adhikari et al., 2018). The yield of fine rice is lower than that of coarse and 
al.

medium rice varieties. To overcome this situation increment of aromatic rice production unit ${ }^{-1}$ area is the only alternative to bring self-sufficiency in aromatic rice through intensive care, management and adoption of new technologies. IRRI has developed AWD (Alternate Wetting and Drying) technology. This system can save 30\% irrigation water, save energy and fuel consumption. AWD requires irrigation when the water level goes down to $15 \mathrm{~cm}$ below the soil surface. SRI (System of rice intensification) is another water saving technology (Stool et al., 2002) where minimum water input with intermittent or saturated soil requires 20$50 \%$ less water (Lek and Yongyod, 1989) than continuous flooding.

In recent years there has been serious concern about long-term adverse effect of continuous and indiscriminate use of inorganic fertilizers on deterioration of soil structure, soil health and environmental pollution (Ghosh and Bhat, 1998). Combined application of manure with inorganic fertilizers increased grain yield and protein content of aromatic rice (Sarkar et al., 2014, Roy et al., 2015; Pal et al., 2016; Biswas et al., 2016 and Marzia et al., 2016). High competitive ability of weeds exerts a serious negative effect on crop production causing significant losses in crop yield. Yield losses due to weed infestation are greater than the combined losses of insect pests and diseases. In Bangladesh, weed infestation reduces the grain yield $70-80 \%$ in Aus rice, $30-40 \%$ for transplanted Aman rice and 22-36\% for modern Boro rice cultivars (Mamun, 1990). In case of aromatic rice grain yield was reduced by $28.16 \%$ in Binadhan-9 in Aman season (Zannat et al., 2014) whereas by $59.82 \%$ in BRRI dhan50 at Boro season (Sinha et al., 2018) due to weed infestation. Herbicides are effective in controlling weeds alone or in combination with hand weeding (Ahmed et al., 2005). Herbicides in combination with hand weeding would help to obtain higher crop yield with less efforts and cost (Prasad and Rafy, 1995; Sathyamoorthy et al., 2004). So, the present study was undertaken to find out the effect of water, nutrient and weed management on the growth, yield and quality of aromatic Bororice (cv. BRRI dhan50).

\section{Materials and Methods}

The experiment was carried out at the Agronomy Field Laboratory of Bangladesh Agricultural University, Mymensingh during January to June 2016 to study the effect of water, nutrient and weed management practices on the performance of aromatic Boro rice. The experimental field was located at $24^{\circ \circ} 75^{\prime} \mathrm{N}$ latitude and $90^{\circ} 50$ 'E longitude at an elevation of $18 \mathrm{~m}$ above the mean sea level. The experimental area belongs to non-calcareous dark grey floodplain soil under the Sonatola soil series of Old Brahmaputra Floodplain in Agro Ecological Zone (AEZ- 9) (UNDP and FAO, 1988). The experimental field was medium high, fairly leveled with well drained soils. The soils of this series are pre-dominantly silty loam, dark grey in color having $\mathrm{pH} 6.5$ and low in organic matter.

The experiment comprised three water management viz. Conventional flood irrigation - $\left(\mathrm{I}_{1}\right)$, AWD (Alternate wetting and drying) - $\left(\mathrm{I}_{2}\right)$ and SRI (System of rice intensification) - $\left(\mathrm{I}_{3}\right)$; two levels of nutrient management viz. Recommended dose of inorganic fertilizer (Urea, TSP, MoP, Gypsum, $\mathrm{ZnSO}_{4}$ @ 250, 120, 120, 100, $10 \mathrm{~kg} \mathrm{ha}{ }^{-1}$ respectively) - $\left(\mathrm{F}_{1}\right)$ and $25 \%$ less than recommended dose of inorganic fertilizer + poultry manure @ $2.5 \mathrm{t} \mathrm{ha}{ }^{-1}-\left(\mathrm{F}_{2}\right)$, and there levels of weed management viz. weedy check (control) - $\left(\mathrm{W}_{0}\right)$,two hand weedings at 20 and 40 (Days after transplanting) DAT - $\left(\mathrm{W}_{1}\right)$ and application of Pre-emergence herbicide (Rifit 33EC) followed by post emergence herbicide (Fast Klin 10WP) $\left(\mathrm{W}_{2}\right)$. The experiment was laid out in a randomized complete block design with three replications. Eachunit plot size was $4.0 \mathrm{~m}$ Ч $2.5 \mathrm{~m}$. The rice var. BRRI 
dhan50 of aromatic Boro rice was used as the test crop. Sprouted seeds were sown in the nursery bed on 13 December 2015 for AWD and continuous flooding practices. In SRI method seeds were sown in 11 January 2016. The experimental plots were fertilized with as per experimental treatments. The entire amounts of triple super phosphate, muriate of potash, gypsum and zinc sulphate and poultry manure were applied at final land preparation. Urea was applied in three equal installments at 15, 30 and 45 DAT. The seedlings were uprooted on 26 January 2016 without causing much mechanical injury to the roots and they were immediately transplanted in the well puddled main fieldat the rate of two seedlings hill ${ }^{-1}$, maintaining row and hill distance of $25 \mathrm{~cm}$ and $15 \mathrm{~cm}$ in AWD and Conventional flooding systems. In case of SRI, single seedling hill ${ }^{-1}$ was used12-day old seedlings whereas conventional and AWD systems 30-day old seedlings were used. AWD is monitored the depth of irrigated water on the field using a 'field water tube' or magic pipe. It involves installation of a perforated pipe in the rice field to allow observation of water level. In this experiment, magic pipe was installed at 10 DAT. After irrigation, the depth of irrigation water will gradually decrease. When the irrigation water has dropped to $15 \mathrm{~cm}$ below the surface of the soil, irrigation was applied with $5 \mathrm{~cm}$ depth water. From one week before to one week after flowering, irrigation water was kept at $5 \mathrm{~cm}$ depth. After flowering, the water level was dropped again to $15 \mathrm{~cm}$ below the surface before irrigation. Water management was the most complicated variable in SRI method where rice crops are kept unflooded during vegetative growth with partial amount of water was applied to keep the soil moist.It was even allowed to dry out for 2 to 4 days during tillering to keep the soil well aerated and to allow better root growth. From panicle initiation (PI) to hard dough stage, a thin layer of water $(2-3 \mathrm{~cm})$ was kept on the plots. Again water was drained out from the plots during ripening stage.

Data on weed population were recorded from each plot at 65 DAT by using 1 $\mathrm{m}^{2}$ quadrate as per method described by Cruz et al. (1986). The weeds inside each quadrate were uprooted and cleaned, separated species-wise and dried first in the sun and then in an electrical oven for 72 hours at a temperature of 80 ${ }^{\circ} \mathrm{C}$ followed by weed dry weight of each plot was recorded by an electrical balance. The crop was harvested on 25 May, 30 May and 06 June 2016, respectively for conventional, AWD and SRI. The grains were cleaned and finally the weight was adjusted to a moisture content of $14 \%$. The straw was sun dried and the yields of grain and straw plot $^{-1}$ were recorded and converted to $t$ ha $^{-1}$. Harvest index indicates the ratio of economic yield (grain yield) to biological yield (grain yield + straw yield) and was calculated by the following formula:

Harvest index (\%)

$$
=\quad \frac{\text { Grain Yield }\left(\mathrm{tha}^{\circ}\right)}{\text { Biological Yield }\left(\mathrm{tha}^{\circ}\right)} \text { Чx } 100 .
$$

Estimation of protein in grains was done by following Micro-Kjeldahl Method. Oven dried rice kernels were then put in polythene bags and kept in desiccators for subsequent chemical analysis for estimation of protein. Protein content (\%) was estimated by Micro-Kjeldahl method (AOAC, 1984) at the Agri-Varsity Humboldt Soil Testing Laboratory of Soil Science Department, Bangladesh Agricultural University, Mymensingh. The recorded data were compiled and tabulated for statistical analysis. Analysis of variance was done with the help of computer package, MSTAT. The mean differences among the treatments were adjudged by Duncan's Multiple Range Test (Gomez and Gomez, 1984). 


\section{Results and Discussion}

\section{Weed dry weight}

There was significant effect on total weed dry weight $\left(\mathrm{g} \mathrm{m}^{-2}\right)$ due to water management (Table 1). The highest total weed dry weight $\left(38.71 \mathrm{~g} \mathrm{~m}^{-2}\right)$ was found in $I_{1}$ (Conventional flood irrigation) followed by AWD (Alternate Wetting and Drying), while the lowest total weed dry weight $\left(33.35 \mathrm{~g} \mathrm{~m}^{-2}\right)$ was found in $\mathrm{I}_{3}$ (SRI) water management (Table 1).There was significant effect on total weed dry weight due to nutrient management (Table 1). Maximum weed dry weight $\left(37.75 \mathrm{~g} \mathrm{~m} \mathrm{~m}^{-2}\right)$ was found in $F_{1}$ (Recommended dose of inorganic fertilizer) and minimum one $\left(33.56 \mathrm{~g} \mathrm{~m}^{-2}\right)$ was found in $\mathrm{F}_{2}(25 \%$ less than recommended dose of inorganic fertilizer + poultry manure @ $2.5 \mathrm{t} \mathrm{ha}^{-1}$ ) nutrient management (Table 1). There was significant effect on total weed dry weight due to weed management (Table 1). At 65 DAT, the maximum total weed dry weight $\left(75.03 \mathrm{~g} \mathrm{~m}^{-2}\right.$ ) was found in $\mathrm{W}_{0}$ (No weeding) followed by two hand weedings at 20 and 40 DAT while the lowest total weed dry weight $\left(8.91 \mathrm{~g} \mathrm{~m}^{-2}\right)$ in $\mathrm{W}_{2}$ (Pre-emergence herbicide Rifit 33EC followed by post emergence herbicide Fast Klin 10WP) weed management (Table 1).Non-significant variation was found on total weed dry weight due to interaction effect of water and nutrient management at 65 DAT (Table 2). However, numerically, the highest weed dry weight (41.43 $\mathrm{g} \mathrm{m}^{-2}$ ) was found in $\mathrm{I}_{1} \mathrm{YF}_{1}$ (Conventional flood irrigation $\mathrm{Y}$ Recommended dose of inorganic fertilizer) and the lowest $\left(31.35 \mathrm{~g} \mathrm{~m}^{-2}\right)$ one was found in $\mathrm{I}_{3} \mathrm{YF}_{2}$ (SRI $\mathrm{Y}$ $25 \%$ less than recommended dose of inorganic fertilizer + poultry manure) treatment (Table 2). Significant variation was found on total weed dry weight due to interaction effect of water and weed management at 65 DAT (Table 3). The highest weed dry weight $\left(80.48 \mathrm{~g} \mathrm{~m}^{-2}\right.$ ) was found in $\mathrm{I}_{1} 4 \mathrm{WW}_{0}$ (Conventional flood irrigation $\mathrm{Y}$ No weeding) followed by $\mathrm{I}_{2} \mathrm{Y} \mathrm{W}_{0}$ (AWD $\mathrm{Y}$ No weeding) and $\mathrm{I}_{3} \mathrm{Y}$ $\mathrm{W}_{0}$ (SRI $\mathrm{Y}$ No weeding) and the lowest $\left(7.66 \mathrm{~g} \mathrm{~m}^{-2}\right)$ one was found on $\mathrm{I}_{3} \mathrm{YW}_{2}$ (SRI Y Pre-emergence herbicide Rifit 33EC followed by post emergence herbicide Fast Klin 10WP) treatment (Table 3).Significant variation was found on total weed dry weight due to interaction effect of nutrient and weed management practices at 65 DAT (Table 4). The highest total weed dry weight $\left(77.74 \mathrm{~g} \mathrm{~m}^{-2}\right)$ was found in $F_{1} 4 W_{0}$ (Recommended dose of inorganic fertilizer $\mathrm{Y}$ No Weeding) and the lowest $\left(8.692 \mathrm{~g} \mathrm{~m}^{-2}\right)$ one was found in $F_{1} \mathrm{YW}_{2}$ (Recommended dose of inorganic fertilizer 4 pre-emergence herbicide Rifit 33EC followed by postemergence herbicide Fast Klin 10WP) treatment (Table 4). Non-significant variation was found on total weed dry weight due to interaction effect of water, nutrient and weed management practices at 65 DAT (Table 5).

\section{Yield and yield components}

Crop characters, yield contributing characters, yield and grain protein content were significantly influenced by water management except panicle length and 1000 -grain weight (Table 1). The tallest plant $(82.56 \mathrm{~cm})$, number of total tillers hill $^{-1}$ (12.25), effective tillers hill ${ }^{-1}$ (11.07) and grains panicle ${ }^{-1}$ (119.99) were found in $\mathrm{I}_{3}$ (SRI method) followed by $\mathrm{I}_{2}$ (AWD) and all parameters showed the lowest values in $\mathrm{I}_{1}$ (conventional flood irrigation) (Table 1). While the highest number of non-effective tillers hill ${ }^{-1}(1.44)$ was recorded in AWD (Alternate wetting and drying) and sterile spikelets panicle ${ }^{-1}$ (16.12) were recorded in $I_{1}$ (conventional flood irrigation) and the lowest values were found in $\mathrm{I}_{3}$ (SRI). The highest grain yield (5.41 t ha-1) was obtained in $\mathrm{I}_{3}$ (SRI) water management followed by $\mathrm{I}_{2}$ (AWD) and the lowest grain yield $\left(4.80 \mathrm{t} \mathrm{ha}^{-1}\right)$ in $\mathrm{I}_{1}$ (Conventional flood irrigation). The increased yield might be due to the highest number of grains panicle ${ }^{-1}$ and number of effective tillers hill ${ }^{-1}$. Straw yield also showed similar trend as that of grain yield. The highest grain protein content (8.54\%) was found in $I_{3}$ 
Effect of Water, Nutrient and Weed Management

(SRI) followed by $I_{2}$ (AWD) and the lowest grain protein content (7.96\%) in $I_{1}$ (conventional flood irrigation). 

Table 1. Effect of water management on crop characters, yield components and yield of aromatic Boro rice (cv. BRRI dhan50)

\begin{tabular}{|c|c|c|c|c|c|c|c|c|c|c|c|c|c|}
\hline Treatments & $\begin{array}{l}\text { Dry } \\
\text { matter } \\
\text { of } \\
\text { weed } \\
\left(\mathrm{g} \mathrm{m}^{-2}\right)\end{array}$ & $\begin{array}{l}\text { Plant } \\
\text { height } \\
\text { (cm) }\end{array}$ & $\begin{array}{l}\text { Total tillers } \\
\text { hill }^{-1} \\
\text { (no.) }\end{array}$ & $\begin{array}{l}\text { Effective } \\
\text { tillers hill }{ }^{-1} \\
\text { (no.) }\end{array}$ & $\begin{array}{l}\text { Non- } \\
\text { effective } \\
\text { tillers hill }{ }^{-1} \\
\text { (no.) }\end{array}$ & $\begin{array}{l}\text { Panicle } \\
\text { length } \\
(\mathrm{cm})\end{array}$ & $\begin{array}{l}\text { Grains } \\
\text { panicle } \\
\text { (no.) }\end{array}$ & $\begin{array}{l}\text { No. of } \\
\text { sterile } \\
\text { spikelets } \\
\text { panicle }^{-1}\end{array}$ & $\begin{array}{l}1000- \\
\text { grain } \\
\text { weight } \\
\text { (g) }\end{array}$ & $\begin{array}{l}\text { Grain } \\
\text { yield } \\
\left(\mathrm{t} \mathrm{ha}^{-1}\right)\end{array}$ & $\begin{array}{l}\text { Straw } \\
\text { yield } \\
\left(\mathrm{t} \mathrm{ha}^{-1}\right)\end{array}$ & $\begin{array}{l}\text { Harvest } \\
\text { index } \\
(\%)\end{array}$ & $\begin{array}{l}\text { Grain } \\
\text { protein } \\
\text { content } \\
(\%)\end{array}$ \\
\hline $\mathrm{I}_{1}$ & $38.71 \mathrm{a}$ & $76.96 b$ & $10.49 \mathrm{~b}$ & $9.08 \mathrm{~b}$ & $1.41 \mathrm{a}$ & 21.36 & $116.11 \mathrm{c}$ & $16.12 \mathrm{a}$ & 18.30 & $4.80 c$ & $5.20 c$ & 48.00 & $7.957 b$ \\
\hline $\mathrm{I}_{2}$ & $34.90 \mathrm{~b}$ & $82.49 a$ & $10.92 b$ & $9.48 \mathrm{~b}$ & $1.44 a$ & 21.64 & $117.03 \mathrm{~b}$ & $14.11 \mathrm{~b}$ & 18.64 & $4.99 \mathrm{~b}$ & $5.46 \mathrm{~b}$ & 47.75 & $8.093 \mathrm{~b}$ \\
\hline $\mathrm{I}_{3}$ & $33.35 c$ & $82.56 a$ & $12.25 a$ & $11.07 \mathrm{a}$ & $1.18 \mathrm{~b}$ & 21.89 & $119.99 a$ & $13.75 c$ & 19.32 & $5.41 \mathrm{a}$ & $6.05 a$ & 47.20 & $8.536 a$ \\
\hline \multicolumn{14}{|c|}{ Nutrient management } \\
\hline $\mathrm{F}_{1}$ & $37.75 a$ & 80.69 & $10.81 \mathrm{~b}$ & $9.40 \mathrm{~b}$ & $1.41 \mathrm{a}$ & 21.52 & $114.40 \mathrm{~b}$ & $15.10 \mathrm{a}$ & $18.41 \mathrm{~b}$ & $4.72 \mathrm{~b}$ & $5.37 \mathrm{~b}$ & 46.78 & $8.00 \mathrm{~b}$ \\
\hline $\mathrm{F}_{2}$ & $33.56 b$ & 80.66 & $11.63 a$ & $10.36 \mathrm{a}$ & $1.28 \mathrm{~b}$ & 21.74 & $117.68 \mathrm{a}$ & $14.22 \mathrm{~b}$ & $19.15 a$ & $5.03 a$ & $5.77 \mathrm{a}$ & 46.57 & $8.26 a$ \\
\hline CV (\%) & 6.44 & 3.62 & 7.62 & 5.17 & 11.84 & 4.37 & 8.19 & 14.46 & 4.94 & 3.86 & 4.00 & 3.48 & 4.18 \\
\hline \multicolumn{14}{|c|}{ Weed management } \\
\hline $\mathrm{W}_{0}$ & $75.03 a$ & $78.81 \mathrm{~b}$ & $9.13 c$ & $8.17 \mathrm{c}$ & $0.96 c$ & $\begin{array}{c}21.22 \\
\mathrm{~b}\end{array}$ & 116.53 & $16.50 \mathrm{a}$ & $18.12 b$ & $2.91 \mathrm{c}$ & $3.80 c$ & $43.36 c$ & $7.92 b$ \\
\hline $\mathrm{W}_{1}$ & $23.03 b$ & $81.46 a$ & $11.62 \mathrm{~b}$ & 9.97b & $1.65 a$ & $21.57 \mathrm{ab}$ & 118.05 & $14.03 \mathrm{~b}$ & $18.50 \mathrm{~b}$ & $5.10 \mathrm{~b}$ & $6.19 b$ & $45.17 \mathrm{~b}$ & $8.12 b$ \\
\hline $\mathrm{W}_{2}$ & $8.91 \mathrm{c}$ & $81.75 a$ & $12.91 \mathrm{a}$ & $11.50 \mathrm{a}$ & $1.41 \mathrm{~b}$ & $22.10 \mathrm{a}$ & 120.55 & $13.46 \mathrm{c}$ & $19.64 a$ & $5.75 a$ & $6.73 a$ & $46.07 a$ & $8.54 a$ \\
\hline CV (\%) & 6.44 & 3.62 & 7.62 & 5.17 & 11.84 & 4.37 & 8.19 & 14.46 & 4.94 & 3.86 & 4.00 & 3.48 & 4.18 \\
\hline
\end{tabular}

In a column, figures with same letter or without letter do not differ significantly (as per DMRT).

$\mathrm{I}_{1}=$ Conventional flood irrigation, $\mathrm{I}_{2}=$ AWD (Alternate Wetting and Drying), $\mathrm{I}_{3}=\mathrm{SRI}$ (System of Rice Intensification), $\mathrm{F}_{1}=\mathrm{Recommended}$

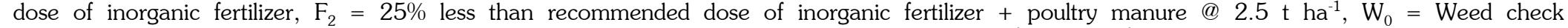
(control), $\mathrm{W}_{1}=$ Two hand weeding at 20 and $40 \mathrm{DAT}, \mathrm{W}_{2}=$ Pre-emergence herbicide (Rifit 33EC) followed by post emergence herbicide (Fast Klin 10WP) 
All yield contributing characters, yield and grain protein content were significantly influenced by nutrient management except plant height and panicle length (Table 1). The highest number of total tillers hill $^{-1}$ (11.63), effective tillers hill ${ }^{-1}$ (10.36), grains panicle ${ }^{-1}(117.68)$ and 1000 -grain weight $(19.15 \mathrm{~g})$ were found in all the parameters showed lower values in $\mathrm{F}_{1}$. The highest number of non-effective tillers hill $^{-1}$ (1.41) and sterile spikelets panicle ${ }^{-1}$ (15.10) were produced in $F_{1}$ nutrient management, while the lowest number of sterile spikelets panicle ${ }^{-1}$ (14.22) in $\mathrm{F}_{2}$ (25\% less than recommended dose of inorganic fertilizer + poultry manure @ 2.5 $\left.\mathrm{t} \mathrm{ha}^{-1}\right)$. Higher grain yield $\left(5.03 \mathrm{t} \mathrm{ha}^{-1}\right)$ and straw yield $\left(5.77 \mathrm{t} \mathrm{ha}^{-1}\right)$ were obtained in $\mathrm{F}_{2}$ (25\% less than recommended dose of inorganic fertilizer + poultry manure@ $@ 2.5 \mathrm{t} \mathrm{ha}^{-1}$ ) nutrient management than that of $\mathrm{F}_{1}$. The increased yield might be due to higher number of grains panicle ${ }^{-1}$, higher 1000 -grain weight and higher number of effective tillers hill ${ }^{-1}$. Application of inorganic fertilizer along with manure greatly influence the grain yield of rice were reported (Islam et al., 2015; Roy et al., 2015; Pal et al., 2016; Biswas et al., 2016 and Sarkar et al., 2016). Table 1 indicates that higher grain protein content (8.26\%) was found in $F_{2}$ compared to $F_{1}$. Integration of poultry manure with chemical fertilizer increased grain protein content was also reported (Sarkar et al., 2014; Biswas et al., 2016 and $\mathrm{Pal}$ et al., 2016)

Crop characters, yield contributing characters, yield and grain protein content were significantly influenced by water management except grains panicle ${ }^{-1}$ (Table 1). The tallest plant $(81.75 \mathrm{~cm})$ was found in $\mathrm{W}_{2}$ (pre-emergence herbicide Rifit 33EC followed by post emergence herbicide Fast Klin 10WP) which was at par with $\mathrm{W}_{1}$ (two hand weeding at 20 and 40 DAT) while the shortest plant $(78.81$ $\mathrm{cm}$ ) was found in $\mathrm{W}_{0}$ (No weeding). The highest number of total tillers hill ${ }^{-1}$ (12.91), effective tillers hill ${ }^{-1}(11.50)$, longest panicle $(22.10 \mathrm{~cm})$ and the highest weight of 1000 -grain $(19.64 \mathrm{~g})$ were obtained in $\mathrm{W}_{2}$ whileall the parameters showed intermediate values in $\mathrm{W}_{1}$ and the corresponding lowest values in $\mathrm{W}_{0}$. The highest number of non-effective tillers hill ${ }^{-1}$ (1.65) was produced in $W_{1}$ while the lowest number of non-effective tillers hill ${ }^{-1}(0.96)$ in $W_{0}$. The highest number of sterile spikelets panicle ${ }^{-1}$ (16.50) was produced in $\mathrm{W}_{0}$ weed management, while the lowest number of sterile spikelets panicle $e^{-1}$ (13.46) in $W_{2}$. Number of grains panicle $^{-1}$ was not significantly influenced by different weed management. Table 1 indicates that numerically the highest number of grains panicle ${ }^{-1}$ (120.55) was produced in $\mathrm{W}_{2}$ while the lowest number of grains panicle ${ }^{-1}(116.53)$ by $\mathrm{W}_{0}$. The highest grain yield $\left(5.75 \mathrm{t} \mathrm{ha}^{-1}\right)$, straw yield $\left(6.73 \mathrm{t} \mathrm{ha}^{-1}\right)$ and harvest index (46.07\%) were produced in $\mathrm{W}_{2}$ followed by $\mathrm{W}_{1}$ while the corresponding lowest values were obtained in $\mathrm{W}_{0}$. Weed infestation reduced $49.39 \%$ grain yield over $\mathrm{W}_{2}$. Similar trend was reported by Sinha et al. (2018) that weed reduced grain yield by $59.83 \%$ in aromatic Boro rice cv. BRRI dhan50. The highest grain protein content (8.54\%) was found in $\mathrm{W}_{2}$ and the lowest grain protein content $(7.92 \%)$ in $\mathrm{W}_{0}$ which was at par with $\mathrm{W}_{1}$.

Table 2 indicates that the highest number of total tillers hill ${ }^{-1}(12.78)$, effective tillers hill- ${ }^{1}$ (11.67), and grain yield $\left(5.19 \mathrm{t} \mathrm{ha}^{-1} \mathrm{t}\right.$ ha $\left.{ }^{-1}\right)$ were obtained in $\mathrm{I}_{3} \mathrm{Y} \mathrm{F}_{2}$ (SRI 4 25\% less than recommended dose of inorganic fertilizer + poultry manure @ $2.5 \mathrm{t} \mathrm{ha}^{-1} \mathrm{t}$ ). Grain yield produced in $\mathrm{I}_{3} \mathrm{Y}_{1} \mathrm{~F}_{1}$ (SRI $\mathrm{Y}$ recommended dose of inorganic fertilizer) was at parinI ${ }_{3} \mathrm{Y} \mathrm{F}_{2}$ (SRI-25\% less than recommended dose of inorganic fertilizer + poultry manure @ $\left.2.5 \mathrm{t} \mathrm{ha}^{-1} \mathrm{t}\right)$ while the corresponding lowest values in $\mathrm{I}_{1} \mathrm{~F}_{1}$ (conventional flood irrigation $\mathrm{Y}$ recommended dose of inorganic fertilizer). 
Table 2. Interaction effect of water and nutrient management on crop characters, yield components and yield aromatic Boro rice (cv. BRRI dhan50)

\begin{tabular}{|c|c|c|c|c|c|c|c|c|c|c|c|c|c|}
\hline $\begin{array}{l}\text { Interaction } \\
\text { (Water Ч } \\
\text { nutrient } \\
\text { management) }\end{array}$ & $\begin{array}{l}\text { Dry } \\
\text { matter } \\
\text { of } \\
\text { weed } \\
\left(\mathrm{g} \mathrm{m}^{-}\right. \\
2 \text { ) }\end{array}$ & $\begin{array}{l}\text { Plant } \\
\text { height } \\
(\mathrm{cm})\end{array}$ & $\begin{array}{l}\text { Total } \\
\text { tillers } \\
\text { hill }^{-1} \\
\text { (no.) }\end{array}$ & $\begin{array}{l}\text { Effective } \\
\text { tillers } \\
\text { hill }^{-1} \\
\text { (no.) }\end{array}$ & $\begin{array}{l}\text { Non- }^{-} \\
\text {effective } \\
\text { tillers hill }{ }^{-1} \\
\text { (no.) }\end{array}$ & $\begin{array}{l}\text { Panicle } \\
\text { length } \\
(\mathrm{cm})\end{array}$ & $\begin{array}{l}\text { Grains } \\
\text { panicle } \\
1 \\
\text { (no.) }\end{array}$ & $\begin{array}{l}\text { No. of } \\
\text { sterile } \\
\text { spikelets } \\
\text { panicle }^{-1}\end{array}$ & $\begin{array}{l}1000- \\
\text { grain } \\
\text { weight } \\
\text { (g) }\end{array}$ & $\begin{array}{l}\text { Grain } \\
\text { yield } \\
\left(\mathrm{t} \mathrm{ha}^{-1}\right)\end{array}$ & $\begin{array}{l}\text { Straw } \\
\text { yield } \\
\left(\mathrm{t} \mathrm{ha}^{-1}\right)\end{array}$ & $\begin{array}{l}\text { Harvest } \\
\text { index } \\
(\%)\end{array}$ & $\begin{array}{l}\text { Grain } \\
\text { protein } \\
\text { content } \\
(\%)\end{array}$ \\
\hline $\mathrm{I}_{1} \mathrm{YF}_{1}$ & 41.43 & 82.59 & $10.15 c$ & $8.64 d$ & 1.52 & 21.22 & 113.40 & 16.89 & 18.07 & $4.76 c$ & 5.01 & 48.72 & 7.58 \\
\hline $\mathrm{I}_{1} \mathrm{YF}_{2}$ & 35.98 & 82.54 & $10.82 b c$ & $9.52 b c$ & 1.29 & 21.50 & 114.82 & 15.35 & 18.54 & $4.92 b$ & 5.44 & 47.49 & 7.93 \\
\hline $\mathrm{I}_{2} \mathrm{UF}_{1}$ & 36.43 & 82.14 & $10.51 b c$ & $9.09 c$ & 1.42 & 21.66 & 115.10 & 14.31 & 18.27 & $4.80 \mathrm{bc}$ & 5.28 & 47.61 & 7.94 \\
\hline $\mathrm{I}_{3} \mathrm{YF}_{2}$ & 31.35 & 76.58 & $12.78 \mathrm{a}$ & $11.67 a$ & 1.09 & 22.10 & 118.26 & 13.38 & 19.77 & $5.19 a$ & 6.24 & 45.40 & 8.59 \\
\hline CV (\%) & 6.44 & 3.62 & 7.62 & 5.17 & 11.84 & 4.37 & 8.19 & 14.46 & 4.94 & 3.86 & 4.00 & 3.48 & 4.18 \\
\hline
\end{tabular}

In a column, figures with same letter or without letter do not differ significantly (as per DMRT).

$\mathrm{I}_{1}=$ Conventional flood irrigation, $\mathrm{I}_{2}=$ AWD (Alternate Wetting and Drying), $\mathrm{I}_{3}=$ SRI (System of Rice Intensification)

$\mathrm{F}_{1}=$ Recommended dose of inorganic fertilizer, $\mathrm{F}_{2}=25 \%$ less than recommended dose of inorganic fertilizer + poultry manure @ $2.5 \mathrm{t}$ ha

Table 3 indicates that the highest number of grains panicle ${ }^{-1}(119.0)$ was produced in $\mathrm{I}_{3} \mathrm{U} \mathrm{W}_{2}$ (SRI $\mathrm{Y}$ pre-emergence herbicide Rifit 33EC followed by post-emergence herbicide Fast Klin 10WP) which was at par to $\mathrm{I}_{2} \mathrm{~W}_{2}$ (AWD pre-emergence herbicide Rifit 33EC followed by post emergence herbicide Fast Klin 10WP). The highest grain yield $\left(6.14 \mathrm{t}^{-1}\right.$ has was produced in $\mathrm{I}_{3} \mathrm{~W}_{2}$ followed by $\mathrm{I}_{3} \mathrm{~W}_{2}$ (SRI two hand weeding at 20 and 40 DAT) $\left(6.14 \mathrm{t} \mathrm{ha}^{-1}\right)$ and the lowest grain yield $\left(2.70 \mathrm{t}\right.$ ha $\left.{ }^{-1}\right)$ in $\mathrm{I}_{1} \mathrm{YW}_{0}$ (conventional flood irrigation $\mathrm{Y}$ no weeding). The highest number of effective tillers hill ${ }^{-1}(11.96)$ was obtained in $F_{2} \Psi W_{2}(25 \%$ less than recommended dose of inorganic fertilizer + poultry manure @ $2.5 \mathrm{t} \mathrm{ha}^{-1} \mathrm{4}$ pre-emergence herbicide Rifit 33EC followed by post-emergence herbicide Fast Klin 10WP) followed by $\mathrm{F}_{1} \mathrm{UW}_{2}$ (recommended dose of inorganic fertilizer preemergence herbicide Rifit 33EC followed by post emergence herbicide Fast Klin 10WP) and lowest one (7.79) in $\mathrm{F}_{1} \mathrm{YW}_{0}$ (recommended dose of inorganic fertilizer no weeding) 
Table 3. Interaction effect of water and weed management on crop characters, yield components and yield of aromatic Boro rice (cv. BRRI dhan50)

\begin{tabular}{|c|c|c|c|c|c|c|c|c|c|c|c|c|c|}
\hline $\begin{array}{l}\text { Interaction } \\
\text { (Water 4 } \\
\text { weed } \\
\text { management) }\end{array}$ & $\begin{array}{l}\text { Dry } \\
\text { matter } \\
\text { of } \\
\text { weed } \\
\left(\mathrm{g} \mathrm{m}^{-2}\right)\end{array}$ & $\begin{array}{l}\text { Plant } \\
\text { height } \\
(\mathrm{cm})\end{array}$ & $\begin{array}{l}\text { Total } \\
\text { tillers } \\
\text { hill }^{-1} \\
\text { (no.) }\end{array}$ & $\begin{array}{l}\text { Effective } \\
\text { tillers } \\
\text { hill }^{-1} \\
\text { (no.) }\end{array}$ & $\begin{array}{l}\text { Non- } \\
\text { effective } \\
\text { tillers } \\
\text { hill }^{-1} \\
\text { (no.) }\end{array}$ & $\begin{array}{l}\text { Panicle } \\
\text { length } \\
(\mathrm{cm})\end{array}$ & $\begin{array}{l}\text { Grains } \\
\text { panicle }{ }^{-1} \\
\text { (no.) }\end{array}$ & $\begin{array}{l}\text { No. of } \\
\text { sterile } \\
\text { spikelets } \\
\text { panicle }^{-1}\end{array}$ & $\begin{array}{l}\text { 1000- } \\
\text { grain } \\
\text { weight } \\
\text { (g) }\end{array}$ & $\begin{array}{l}\text { Grain } \\
\text { yield } \\
\left(\mathrm{t} \mathrm{ha}{ }^{-1}\right)\end{array}$ & $\begin{array}{l}\text { Straw } \\
\text { yield } \\
\left(\mathrm{t} \mathrm{ha}{ }^{-1}\right)\end{array}$ & $\begin{array}{l}\text { Harvest } \\
\text { index } \\
(\%)\end{array}$ & $\begin{array}{l}\text { Grain } \\
\text { protein } \\
\text { content } \\
(\%)\end{array}$ \\
\hline $\mathrm{I}_{1} \cup \mathrm{W}_{0}$ & $80.48 a$ & 81.47 & 8.39 & 7.40 & $0.99 \mathrm{~d}$ & 20.87 & 116.40de & $20.10 \mathrm{a}$ & 17.71 & $2.70 e$ & 3.45 & 43.90 & 7.35 \\
\hline $\mathrm{I}_{1} \mathrm{Y} \mathrm{W}_{1}$ & $25.80 c$ & 83.65 & 10.88 & 9.14 & $1.74 \mathrm{ab}$ & 21.32 & $117.00 \mathrm{~cd}$ & $14.53 \mathrm{bc}$ & 18.12 & $5.07 \mathrm{c}$ & 5.81 & 46.59 & 7.82 \\
\hline $\mathrm{I}_{1} \mathrm{Ч} \mathrm{W}_{2}$ & $9.832 e$ & 82.57 & 12.20 & 10.70 & $1.50 \mathrm{~b}$ & 21.90 & $117.93 b$ & $13.73 \mathrm{~cd}$ & 19.08 & $5.43 b c$ & 6.35 & 46.09 & 8.11 \\
\hline $\mathrm{I}_{2} Ч \mathrm{~W}_{0}$ & $73.46 b$ & 81.15 & 8.71 & 7.79 & $0.91 e$ & 21.25 & $116.58 d$ & $14.85 b$ & 17.92 & 2.83de & 3.73 & 43.14 & 7.83 \\
\hline $\mathrm{I}_{2} \mathrm{Y} \quad \mathrm{W}_{1}$ & $22.00 \mathrm{~d}$ & 83.08 & 11.47 & 9.54 & $1.93 a$ & 21.60 & $116.86 \mathrm{~d}$ & $13.96 \mathrm{bd}$ & 18.40 & $5.41 b c$ & 6.19 & 46.63 & 7.99 \\
\hline $\mathrm{I}_{2} \mathrm{Y} \quad \mathrm{W}_{2}$ & $9.236 e$ & 83.24 & 12.57 & 11.11 & $1.47 \mathrm{~b}$ & 22.08 & 118.63ab & $13.53 \mathrm{~cd}$ & 19.60 & $5.69 b$ & 6.48 & 46.75 & 8.46 \\
\hline $\mathrm{I}_{3}$ Ч $\mathrm{W}_{0}$ & $71.10 \mathrm{~b}$ & 73.81 & 10.30 & 9.31 & $0.99 \mathrm{~d}$ & 21.55 & $117.61 b c$ & $14.56 \mathrm{bc}$ & 18.74 & $3.21 \mathrm{~d}$ & 4.20 & 43.34 & 8.28 \\
\hline $\mathrm{I}_{3} \mathrm{Ч} \mathrm{W}_{1}$ & $21.28 \mathrm{~d}$ & 78.51 & 12.50 & 11.23 & $1.28 \mathrm{c}$ & 21.80 & $117.28 \mathrm{c}$ & $13.59 \mathrm{~cd}$ & 19.00 & $5.69 b$ & 6.58 & 46.37 & 8.46 \\
\hline $\mathrm{I}_{3} \mathrm{Y} \quad \mathrm{W}_{2}$ & $7.662 e$ & 78.57 & 13.95 & 12.68 & $1.27 \mathrm{c}$ & 22.31 & $119.0 \mathrm{a}$ & $13.11 \mathrm{~d}$ & 20.24 & $6.14 a$ & 7.36 & 45.48 & 8.87 \\
\hline CV (\%) & 6.44 & 3.62 & 7.62 & 5.17 & 11.84 & 4.37 & 8.19 & 14.46 & 4.94 & 3.86 & 4.00 & 3.48 & 4.18 \\
\hline
\end{tabular}

In a column, figures with same letter or without letter do not differ significantly (as per DMRT).

$\mathrm{I}_{1}=$ Conventional flood irrigation, $\mathrm{I}_{2}=\mathrm{AWD}$ (Alternate Wetting and Drying), $\mathrm{I}_{3}=$ SRI (System of Rice Intensification), $\mathrm{W}_{0}=\mathrm{Weed}_{\mathrm{c}}$ check (control),

$\mathrm{W}_{1}=$ Two hand weeding at 20 and 40 DAT, $\mathrm{W}_{2}=$ Pre-emergence herbicide (Rifit 33EC) followed by post emergence herbicide (Fast Klin 10WP) 

Table 4. Interaction effect of nutrient and weed management on crop characters, yield components and yield of aromatic Boro rice (cv. BRRI dhan50)

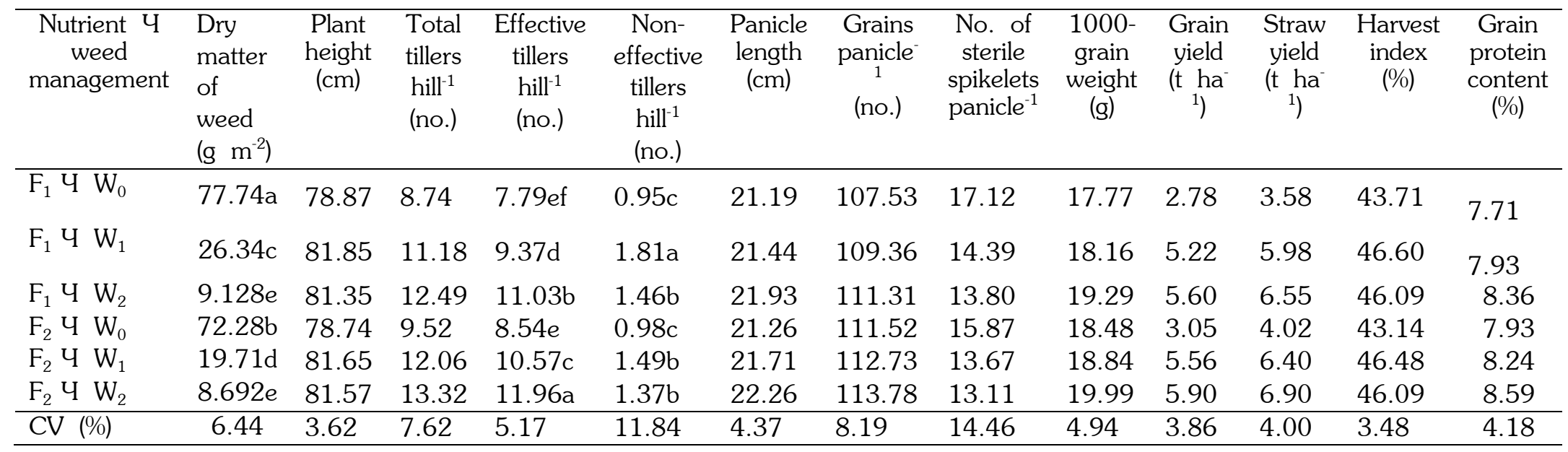

In a column, figures with same letter or without letter do not differ significantly (as per DMRT).

$\mathrm{F}_{1}=$ Recommended dose of inorganic fertilizer, $\mathrm{F}_{2}=25 \%$ less than recommended dose of inorganic fertilizer + poultry manure @ $2.5 \mathrm{t}$ ha ${ }^{-1}$ $\mathrm{W}_{0}=$ Weed check (control), $\mathrm{W}_{1}=$ Two hand weeding at 20 and 40 DAT, $\mathrm{W}_{2}=$ Pre-emergence herbicide (Rifit 33EC) followed by post emergence herbicide (Fast Klin 10WP)

Table 5 indicates that the highest number of total tillers hill ${ }^{-1}$ (14.58), effective tillers hill ${ }^{-1}$ (13.40) and grains panicle $e^{-1}(118.65)$ were obtained in $\mathrm{I}_{3} \mathrm{YF}_{2} 4 \mathrm{WW}_{2}$ (SRI 25\% less than recommended dose of inorganic fertilizer + poultry manure @ $2.5 \mathrm{t}$ ha ${ }^{-1}$ preemergence herbicide Rifit 33EC followed by post emergence herbicide Fast Klin 10WP) treatment while the corresponding

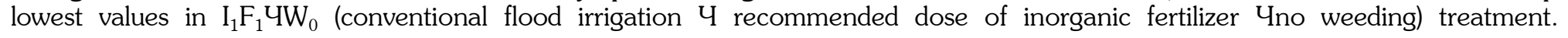



Table 5. Interaction effect of water, nutrient and weed management on crop characters, yield components and yield of aromatic Boro rice (cv. BRRI dhan50)

\begin{tabular}{|c|c|c|c|c|c|c|c|c|c|c|c|c|c|}
\hline $\begin{array}{l}\text { Interaction } \\
\text { (Water Y } \\
\text { nutrient } 4 \\
\text { weed } \\
\text { management) }\end{array}$ & $\begin{array}{l}\text { Dry } \\
\text { matter } \\
\text { of } \\
\text { weed } \\
\left(\mathrm{g} \mathrm{m}^{-2}\right)\end{array}$ & $\begin{array}{l}\text { Plant } \\
\text { height } \\
(\mathrm{cm})\end{array}$ & $\begin{array}{l}\text { Total } \\
\text { tillers hill }{ }^{-1} \\
\text { (no.) }\end{array}$ & $\begin{array}{l}\text { Effective } \\
\text { tillers } \\
\text { hill }^{-1} \\
\text { (no.) }\end{array}$ & $\begin{array}{l}\text { Non- } \\
\text { effective } \\
\text { tillers } \\
\text { hill }^{-1} \\
\text { (no.) }\end{array}$ & $\begin{array}{l}\text { Panicle } \\
\text { length } \\
\text { (cm) }\end{array}$ & $\begin{array}{l}\text { Grains } \\
\text { panicle } \\
\text { (no.) }\end{array}$ & $\begin{array}{l}\text { No. of } \\
\text { sterile } \\
\text { spikelets } \\
\text { panicle }\end{array}$ & $\begin{array}{l}\text { 1000- } \\
\text { grain } \\
\text { weight } \\
\text { (g) }\end{array}$ & $\begin{array}{l}\text { Grain } \\
\text { yield } \\
\left(\mathrm{t} \mathrm{ha} \mathrm{ha}^{-1}\right)\end{array}$ & $\begin{array}{l}\text { Straw } \\
\text { yield } \\
(\mathrm{t} \mathrm{ha} \\
\left.{ }_{1}\right)\end{array}$ & $\begin{array}{l}\text { Harvest } \\
\text { index } \\
(\%)\end{array}$ & $\begin{array}{l}\text { Grain } \\
\text { protein } \\
\text { content } \\
(\%)\end{array}$ \\
\hline $\mathrm{I}_{1}$ Ч $\mathrm{F}_{1}$ Ч $\mathrm{W}_{0}$ & 83.30 & 81.61 & $8.11 \mathrm{f}$ & $7.10 \mathrm{i}$ & 1.01 & 20.56 & $114.17 \mathrm{fg}$ & 21.43 & 17.49 & $2.55 \mathrm{~h}$ & 3.25 & 43.96 & 7.23 \\
\hline $\mathrm{I}_{1}$ Ч $\mathrm{F}_{1}$ Ч $\mathrm{W}_{1}$ & 31.04 & 83.21 & $10.4 d$ & $8.49 \mathrm{fg}$ & 1.91 & 21.21 & 117.17bcde & 15.19 & 17.98 & $4.78 e$ & 5.43 & 46.81 & 7.58 \\
\hline $\mathrm{I}_{1} \mathrm{Y}^{\mathrm{F}_{1}} \mathrm{Y} \quad \mathrm{W}_{2}$ & 9.960 & 82.94 & $11.98 \mathrm{c}$ & $10.33 \mathrm{cde}$ & 1.65 & 21.89 & 117.93ab & 14.05 & 18.74 & $5.25 \mathrm{de}$ & 6.23 & 45.73 & 7.93 \\
\hline $\mathrm{I}_{1} \cup \mathrm{F}_{2} \mathrm{Y} \mathrm{W}_{0}$ & 77.67 & 81.33 & 8.67 ef & $7.70 \mathrm{~g}$ & 0.97 & 21.18 & $117.70 \mathrm{~b}$ & 18.76 & 17.93 & $2.89 \mathrm{~g}$ & 3.66 & 44.12 & 7.47 \\
\hline $\mathrm{I}_{1}$ Ч $\mathrm{F}_{2}$ Ч $\mathrm{W}_{1}$ & 20.56 & 84.09 & $11.37 \mathrm{~cd}$ & 9.80de & 1.57 & 21.42 & $117.83 \mathrm{abc}$ & 13.87 & 18.25 & $5.36 \mathrm{cde}$ & 6.19 & 46.40 & 8.05 \\
\hline $\mathrm{I}_{1} \mathrm{Y} \mathrm{F}_{2}$ Ч $\mathrm{W}_{2}$ & 9.703 & 82.20 & $12.42 \mathrm{~b}$ & $11.07 \mathrm{bcde}$ & 1.35 & 21.90 & 117.93ab & 13.41 & 19.42 & $5.62 b c$ & 6.47 & 46.48 & 8.28 \\
\hline $\mathrm{I}_{2} \mathrm{Y} \mathrm{F}_{1} \mathrm{Y} \mathrm{W}_{0}$ & 75.52 & 82.13 & 8.34efg & 7.42gh & 0.93 & 21.59 & $115.10 \mathrm{f}$ & 15.02 & 17.50 & $2.72 \mathrm{gh}$ & 3.43 & 44.22 & 7.60 \\
\hline $\mathrm{I}_{2}$ Ч $\mathrm{F}_{1}$ Ч $\mathrm{W}_{1}$ & 24.03 & 82.28 & $11.01 \mathrm{cde}$ & 9.03defg & 1.98 & 21.48 & 115.63def & 14.18 & 18.00 & $5.29 \mathrm{~d}$ & 6.07 & 46.56 & 7.82 \\
\hline $\mathrm{I}_{2} \mathrm{U}_{\mathrm{I}} \mathrm{F}_{1} \mathrm{Ч} \mathrm{W}_{2}$ & 9.733 & 82.00 & $12.18 \mathrm{bc}$ & $10.82 \mathrm{c}$ & 1.37 & 21.92 & 116.50cde & 13.71 & 19.31 & $5.49 \mathrm{~cd}$ & 6.35 & 46.36 & 8.40 \\
\hline $\mathrm{I}_{2} \mathrm{\cup} \mathrm{F}_{2} \mathrm{\cup} \mathrm{W}_{0}$ & 71.39 & 80.16 & $9.07 e$ & $8.17 \mathrm{fgh}$ & 0.90 & 20.91 & $116.00 \mathrm{de}$ & 14.67 & 18.34 & 3.00fgh & 4.04 & 42.61 & 8.05 \\
\hline $\mathrm{I}_{2} \mathrm{Ч} \mathrm{F}_{2}$ Ч $\mathrm{W}_{1}$ & 19.96 & 83.88 & $11.93 c$ & $10.05 \mathrm{~d}$ & 1.88 & 21.73 & $117.00 \mathrm{c}$ & 13.74 & 18.79 & $5.52 \mathrm{c}$ & 6.30 & 46.70 & 8.17 \\
\hline $\mathrm{I}_{2} \mathrm{Y} \mathrm{F}{ }_{2} \mathrm{Y} \mathrm{W}_{2}$ & 8.740 & 84.48 & $12.96 \mathrm{abc}$ & $11.40 \mathrm{bcd}$ & 1.56 & 22.24 & 117.77abd & 13.35 & 19.88 & $5.88 b$ & 6.60 & 47.12 & 8.52 \\
\hline $\mathrm{I}_{3} \mathrm{Y}^{\mathrm{F}_{1}} \mathrm{Y} \quad \mathrm{W}_{0}$ & 74.40 & 72.88 & 9.77de & $8.85 f$ & 0.92 & 21.42 & $116.33 d$ & 14.92 & 18.31 & $3.15 \mathrm{fg}$ & 4.06 & 43.68 & 8.28 \\
\hline $\mathrm{I}_{3}$ Ч $\mathrm{F}_{1}$ Ч $\mathrm{W}_{1}$ & 23.95 & 80.05 & $12.13 b c$ & $10.60 \mathrm{~cd}$ & 1.53 & 21.63 & $117.30 \mathrm{bcd}$ & 13.79 & 18.50 & $5.59 \mathrm{bc}$ & 6.43 & 46.50 & 8.40 \\
\hline $\mathrm{I}_{3}$ Ч $\mathrm{F}_{1}$ Ч $\mathrm{W}_{2}$ & 7.690 & 79.10 & $13.32 \mathrm{ab}$ & $11.95 b$ & 1.36 & 21.98 & $117.50 \mathrm{bc}$ & 13.64 & 19.81 & 6.07ab & 7.08 & 46.15 & 8.75 \\
\hline $\mathrm{I}_{3} Ч \mathrm{~F}_{2} \mathrm{Y}$ & 67.79 & 74.74 & $10.83 \mathrm{cdef}$ & $9.77 \mathrm{def}$ & 1.07 & 21.68 & $116.87 \mathrm{~cd}$ & 14.19 & 19.16 & $3.27 f$ & 4.35 & 42.91 & 8.28 \\
\hline $\mathrm{I}_{3} \mathrm{Y} \quad \mathrm{F}_{2} \mathrm{Y} \quad \mathrm{W}_{1}$ & 18.62 & 76.97 & 12.87abcd & $11.85 b c$ & 1.02 & 21.98 & $117.87 \mathrm{ab}$ & 13.39 & 19.49 & $5.80 \mathrm{~b}$ & 6.72 & 46.32 & 8.52 \\
\hline $\mathrm{I}_{3} \cup \mathrm{F}_{2}$ Ч $\mathrm{W}_{2}$ & 7.634 & 78.04 & $14.58 \mathrm{a}$ & $13.40 \mathrm{a}$ & 1.18 & 22.64 & $118.65 a$ & 12.57 & 20.67 & $6.20 \mathrm{a}$ & 7.64 & 44.79 & 8.98 \\
\hline CV (\%) & 6.44 & 3.62 & 7.62 & 5.17 & 11.84 & 4.37 & 8.19 & 14.46 & 4.94 & 3.86 & 4.00 & 3.48 & 4.18 \\
\hline
\end{tabular}

In a column, figures with same letter or without letter do not differ significantly (as per DMRT).

$\mathrm{I}_{1}=$ Conventional flood irrigation, $\mathrm{I}_{2}=$ AWD (Alternate Wetting and Drying), $\mathrm{I}_{3}=$ SRI (System of Rice Intensification)

$\mathrm{F}_{1}=$ Recommended dose of inorganic fertilizer, $\mathrm{F}_{2}=25 \%$ less than recommended dose of inorganic fertilizer + poultry manure @ $2.5 \mathrm{t}$ ha ${ }^{-1}$ $\mathrm{W}_{0}=$ Weed check (control), $\mathrm{W}_{1}=$ Two hand weeding at 20 and 40 DAT, $\mathrm{W}_{2}=$ Pre-emergence herbicide (Rifit 33EC) followed by post emergence herbicide (Fast Klin 10WP) 

The highest grain yield $\left(6.20 \mathrm{t} \mathrm{ha}{ }^{-1}\right)$ was obtained in $\mathrm{I}_{3} \mathrm{UF}_{2} \mathrm{UW}_{2}$ followed by $\mathrm{I}_{3} \mathrm{UF}_{2} \mathrm{YW}_{1}$ while the lowest grain yield $\left(2.55 \mathrm{t}\right.$ ha $\left.{ }^{-1}\right)$ was found in $\mathrm{I}_{1} \mathrm{YF}_{1} \Psi_{0}$. Grain protein content was not statistically significant (Table 5).

A functional relationship was observed between dry matter weight of weeds (at 65 DAT) and grain yield of aromatic Boro rice (cv. BRRI dhan50). A negative linear relationship between weed dry weight (at 65 DAT) and grain yield of aromatic Boro rice was observed, indicating that higher the weed dry matter value lower the grain yield (Figure 1). The functional relationship adequately described by regression equation $Y=-0.0445 x+6.2706\left(R^{2}=0.9714\right)$. The functional relationship indicates that $97 \%$ of the variation in grain yield could be explained from the variation in weed dry matter production at 65 DAT. This finding is in agreement with that of Sinha et al. (2018) who reported that $89 \%$ of Boro rice (cv. BRRI dhan50) yield could be explained by the functional relationship of weed dry matter production at 65 DAT while Islam et al. (2015) reported that $80 \%$ of the variation in grain yield could be explained from the variation in weed dry matter production at 60 DAT in BRRI dhan49.

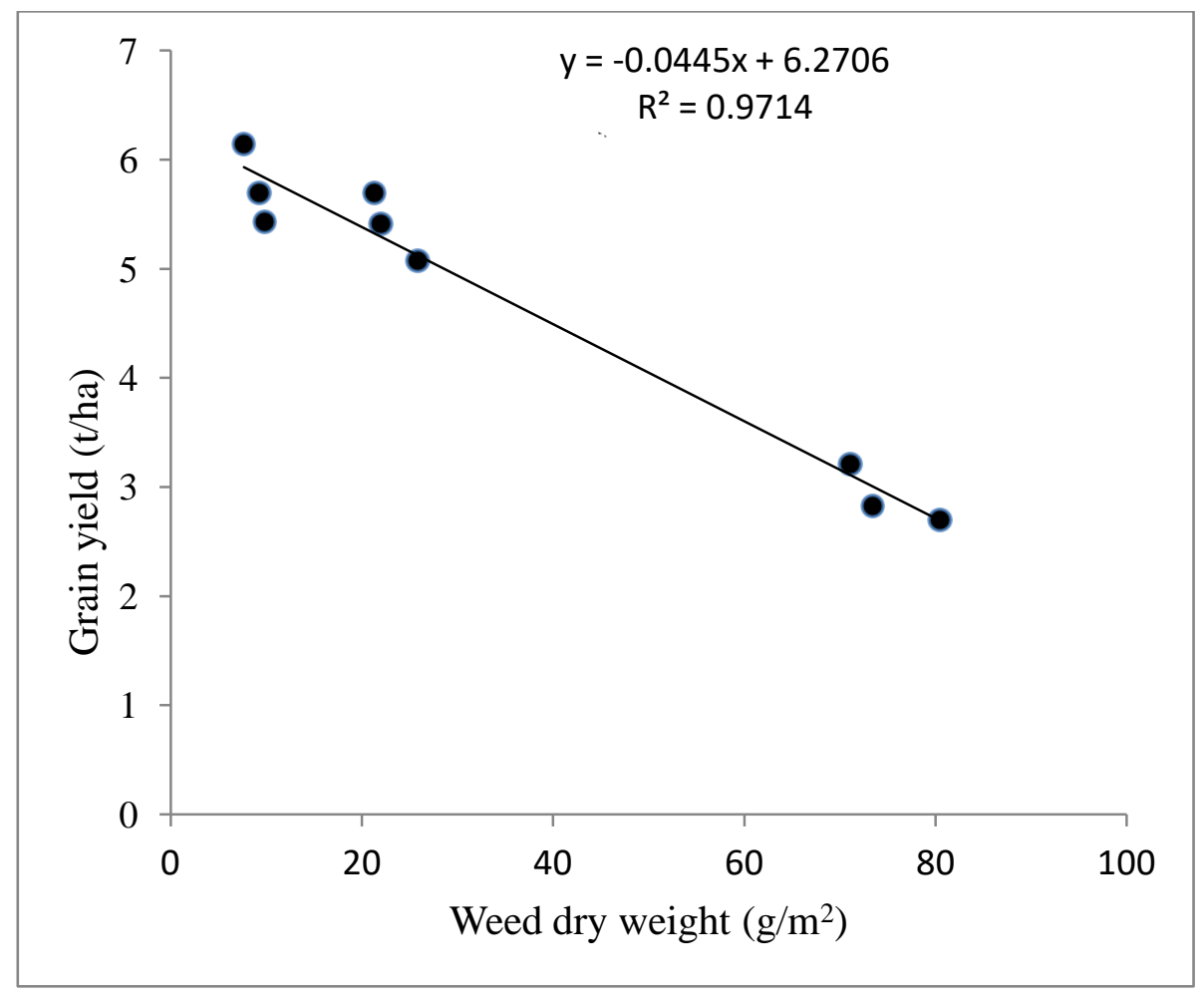

\section{Conclusion}

The highest number of effective tillers hill ${ }^{-1}$,grains panicle ${ }^{-1}$, grain yield and protein (\%) in grain was found in SRI water management along with 25\% less than recommended dose of inorganic fertilizer + poultry manure @ $2.5 \mathrm{t} \mathrm{ha}{ }^{-1}$ and application of pre-emergence herbicide followed by post emergence herbicide. Therefore, it could be concluded that SRI method along with application of $25 \%$ less than recommended dose of inorganic fertilizer + poultry@ 2.5 tha $^{-1}$ and pre-emergence herbicide (Rifit 33EC) followed by post-emergence herbicide 
(Fast Klin 10WP) may be recommended for higher grain yield and quality in terms of grain protein content in aromatic Boro rice cv. BRRI dhan 50.

\section{Acknowledgement}

The financial assistance of Ministry of Science and Technology, Gout. of the People's Republic of Bangladesh, to conduct the research project is thankfully acknowledged.

\section{References}

Adhikari, A., M.A.R. Sarkar, S.K. Paul and K.K. Saha. 2018. Impact of nutrient management on the yield performance of some aromatic fine rice (Oryza sativa L.) varieties in Boro season. Arch. Agr. Environ. Sci. 3(3): 245251.

Ahmed, G.J.U., M.K.A. Bhuiyan, C.R. Riches, M. Mortimer and D. Jhonson. 2005. Farmer's participatory studies of integrated weed management system for intensified lowland. Proceeding of the 8th Biennial Agronomy Convention, Bangladesh Agron. J. 31-32.

AOAC. 1984. Official Methods of Analysis. Association of Official Agricultural Chemists. Washington, D. C. pp. 121-135.

Biswas, T., S.K. Paul, M.A.R. Sarkar and S.K. Sarkar. 2016. Integrated use of poultry manure with prilled urea and urea super granules for improving yield and protein content of aromatic rice (cv. BRRI dhan50). Prog. Agric. 27(2): 86-93.

Cruz, E.D., K. Moody and M.B.D. Ramos. 1986. Reducinguriability in sampling weeds in upland rice (Oryza sativa). Philipp. J. Weed Sci. 13: 56-59.

Ghosh, B.C. and R. Bhat. 1998. Environment hazards of nitrogen loading in wetland rice fields. Environ. Pollut. 102: 123-126.

Gomez, K.A. and A.A. Gomez. 1984. Statistical Procedure for Agricultural Research. Intl. Rice Res. Inst., Philippines, John Wiley and Sons. New York, Chichester, Brisbane, Toronto, Singapore.680p.

Islam, S.M.M., S. K. Paul and M.A.R. Sarkar. 2015. Effect of weeding regime and integrated nutrient management on yield contributing characters and yield of BRRI dhan49. J. Crop Weed. 11 (Special Issue): 193-197.

Lek, P. and S. Yongyod. 1989. Water management for wet and dry season rice growing in irrigated area. Irrigation station 2-10. Paper for 1999 Pathunthani. Rice Research Center Annual Seminar.

Mamun, A.A. 1990. Weeds and their control: A review of weed research in Bangladesh. Agricultural and rural development in Bangladesh.Japan Intl. Co- operation Agency, Dhaka, Bangladesh. JSARD. 19: 45-72.

Marzia, R., M.A.R. Sarkar and S.K. Paul. 2016. Effect of row management and integrated nutrient management on the yield of aromatic fine rice (cv. BRRI dhan34).Int. J. Plant Soil Sci. 13(5): 1-8.

Pal, S., S.K Paul, M.A.R. Sarkar and D.R. Gupta. 2016. Response on yield and protein content of fine aromatic rice varieties to integrated use of cowdung and inorganic fertilizers. J. Crop Weed. 12(1): 01-06. 
al.

Prasad, K. and A. Raffy. 1995. Effect of integrated weed management on weed growth nutrient uptake, economics and energetics in rainfed upland rice (Oryza sativa). Indian J. Agril. Sci. 65(4): 260-264.

Roy, B., M.A.R. Sarkar and S.K. Paul. 2015. Effect of integrated nutrient management in Boro rice cultivation. SAARC J. Agri. 13(2): 131-140.

Sarkar, M.A.R., S.K. Paul and U. Paul. 2016. Effect of plant nutrient and weed management in direct wet seeded Boro rice. Prog. Agric. 27(4): 466-472.

Sarkar, S.K., M.A.R. Sarkar, N. Islam and S.K. Paul. 2014. Yield and quality of aromatic fine rice as affected by variety and nutrient management. $\mathrm{J}$. Bangladesh Agril. Univ. 12(2): 279-284.

Sathyamoorthy, N.K., S. Mahendran, R. Babu and T. Ragavan. 2004. Effect of intgrated weed management practices on total weed dry weight, nutrient removal of weeds in rice-rice wet seedbed system. J. Agron. 3(4): 263267.

Sinha, T., S.K. Paul. and M.A.R. Sarkar. 2018. Effect of age of seedlings at staggered transplanting and weed management on the growth and yield of aromatic Boro rice (cv. BRRI dhan50). J. Bangladesh Agril. Univ. 16(1): 5-11.

Stool, W.A., N. Uphoff and A. Kassam. 2002. A review of agricultural research issues raised by the System of Rice Intensification (SRI) from Madagascar: Opportunities for improving farming systems for resource-poor farmers. Agril. Sys. 71(3): 249-274.

UNDP and FAO. 1988. Land Resources Appraisal of Bangladesh for Agricultural Development. Report 2. Agroecological Regions of Bangladesh. Bangladesh Agril. Res. Coun., Dhaka-1207. pp.212-221.

Zannat, S.T., S.K. Paul and M.A. Salam. 2014. Effect of weeding regime and nitrogen management on the performance of transplant aromatic Aman rice (cv. Binadhan-9). Bangladesh J. Seed Sci. Tech. 18(1 \& 2): 29-34. 